\title{
Análisis de puntos críticos en la cadena de suministro del PET postconsumo en Argentina
}

\author{
Estela Mónica López Sardi' y Juan José Talarico Obónii
}

\section{Resumen}

El reciclado es una de las mejores soluciones para el problema del aumento de la generación de residuos sólidos, ya que contribuye a minimizar el impacto que la industria y el consumo producen en el ambiente. Una de las industrias de reciclado que más ha evolucionado en los últimos años es la del PET.

En el transcurso de un estudio integral sobre reciclado del PET, hemos detectado que el principal problema que enfrentan las empresas dedicadas a esta actividad en Argentina es la recuperación del PET usado. Esto se debe a la existencia de una serie de puntos críticos que afectan el correcto abastecimiento de las industrias recicladoras.

La investigación se realizó aplicando diferentes metodologías: consulta a fuentes primarias a través de entrevistas personales o por e-mail; consulta de fuentes secundarias tales como bibliografía y otros documentos y mediante el uso de software para la simulación de procesos.

El estudio permitió establecer que las principales amenazas para el negocio del PET post consumo en Argentina están dadas por la economía informal, las distancias entre los puntos de generación y las plantas de producción, la dificultad para el acopio y la falta de legislación y normativa que regule adecuadamente la actividad.

Los resultados de la investigación revelan la necesidad de emprender acciones multisectoriales destinadas a optimizar el negocio, basadas en el compromiso proactivo de los ciudadanos, el aporte creativo de las empresas y la acción ambiental sostenida desde las instancias gubernamentales.

Palabras clave: recuperación de PET, reciclado de PET, logística inversa.

Profesora Titular de Química general. Directora de Proyectos de Investigación en Ingeniería Sustentable, Facultad de Ingeniería, Universidad de Palermo.

ii Estudiante de Ingeniería Industrial. Investigador del proyecto "Optimización de rendimiento económico de procesos de reciclado". Facultad de Ingeniería, Universidad de Palermo. 


\section{Abstract}

Recycling is one of the best solutions to the solid waste problem. This kind of actions helps to minimize the environmental impact of industrial activities and consumption. One of the recycling industries that has evolved more in recent years is that of PET.

In the course of a study about PET recycling, we found that one of the main problems faced by this kind of companies in Argentina is the logistics of recovery of used PET. This is due to the existence of a number of critical points affecting the proper supply of the recycling industries.

The research was conducted using different methodologies: consulting primary sources through personal interviews or by e-mail; consultation of secondary sources such as literature and other documents and simulation and modeling of processes by using software.

The study established that the main problems for the business are: informal mechanisms of recovery, long distances between the points of generation and the production facilities, the difficulty for recollection and the lack of laws and regulations.

The research results reveal the need to undertake multi - sectorial action to optimize the business, based on the proactive engagement of citizens, the creative contribution of the companies and the sustained environmental action from government agencies.

Key words: PET recovery, recycled PET, reverse logistics. 


\section{Introducción}

Los residuos constituyen un problema para la sociedad actual, sobre todo en las grandes urbes. La generación excesiva de residuos sólidos urbanos (RSU) junto con el ineficiente manejo que se hace de los mismos, acarrea problemas ambientales, sociales, políticos y daño a la salud pública. Según la Secretaría de Ambiente y Desarrollo Sustentable de la Nación, en Argentina se generan 12.325.000 toneladas al año de residuos sólidos urbanos. Antes de convertirse en basura los residuos han sido materias primas. Uno de los pilares actuales en la gestión de RSU es el triángulo de las 3 R: reducir, reciclar y reutilizar (Junichiro, 2004).

El PET o polietilentereftalato, es un polímero que se obtiene mediante una reacción de policondensación entre el ácido tereftálico y el etilenglicol. Pertenece al grupo de materiales sintéticos denominados poliésteres. El PET puede ser degradado mediante un proceso químico o un proceso natural. Su tiempo de vida media de 500 años cuando queda librado a la degradación natural (Crawford et al., 2013). El método de degradación química permite reciclar el material para un nuevo producto o para la obtención de combustibles (Pilato et al., 2010).

Existen diferentes grados de PET. Los que presentan menor masa molecular se denominan grado fibra, los de masa molecular media, grado película y los de mayor masa molecular, grado ingeniería. Este polímero no se estira y no es afectado por ácidos ni gases atmosféricos, es resistente al calor y absorbe poca cantidad de agua, forma fibras fuertes y flexibles y también películas. Su punto de fusión es alto, lo que facilita su planchado, es resistente al ataque de polillas, bacterias y hongos. Es procesable por soplado, inyección y extrusión. Es apto para producir botellas, películas, láminas, planchas y piezas. Presenta buena barrera al $\mathrm{CO} 2$ y aceptable barrera al $\mathrm{O} 2$ y a la humedad. Es bio - orientable, cristalizable, esterilizable, liviano, transparente y brillante. Altamente reciclable. (Sancho Martín, 2015).

Las especificaciones técnicas del PET lo transforman en un material idóneo para la fabricación de envases. Entre los requisitos que actualmente debe cumplir un envase podemos destacar: adecuada protección del producto, fácil acopio y transporte, ser de bajo costo y ser reciclable. El costo comparativo del PET es algo superior al de otros polímeros plásticos como el polietileno y el polipropileno pero sus propiedades lo transforman en el elegido a la hora de envasar o embotellar bebidas y alimentos. 
En la Tabla 1 se describen otras ventajas del PET que han contribuido a facilitar su inserción cada vez mayor en el mercado de los envases (Blasco, 2015).

\begin{tabular}{|l|l|}
\hline $\begin{array}{l}\text { Evolución en el tiempo } \\
\text { del peso del envase }\end{array}$ & $\begin{array}{l}\text { Los envases de refrescos de PET disminuyeron su peso en } \\
44 \% \text { de } 1980 \text { a la fecha. Los envases de agua y de aceite } \\
\text { disminuyeron su peso en un 37\% en igual lapso de tiempo. }\end{array}$ \\
\hline Optimización de diseño & $\begin{array}{l}\text { Reducción de la boca del envase en un } 28 \% \text { de su peso. } \\
\text { Desaparición de la base cup, sustitución por fondo petaloidal. }\end{array}$ \\
\hline $\begin{array}{l}\text { Homogenización de } \\
\text { materiales }\end{array}$ & $\begin{array}{l}\text { Ya es posible confeccionar tapones, etiquetas, bandejas y film } \\
\text { de PET. }\end{array}$ \\
\hline Ventajas energéticas & $\begin{array}{l}\text { Balance energético favorable frente a otros materiales } \\
\text { cuyos puntos de fusión, reblandecimiento y corte son muy } \\
\text { superiores. } \\
\text { Posibilidad de introducir la etapa de soplado de las preformas } \\
\text { dentro de la línea de envasado del producto. }\end{array}$ \\
\hline Ventajas Logísticas & $\begin{array}{l}\text { Permite transportar un 60\% más de contenido y un } 80 \% \\
\text { menos de envase por camión que en el caso del vidrio. } \\
\text { Permite la fácil manipulación del producto envasado en los } \\
\text { almacenes mayoristas, distribuidores y despachos al público. }\end{array}$ \\
\hline
\end{tabular}

Tabla 1.- Ventajas tecnológicas y logísticas del PET.

El PET post consumo puede ser reciclado obteniendo R-PET, destinado principalmente a la producción de fibras textiles utilizables para la confección de mantas, prendas de vestir, alfombras, calzado, cuerdas, cepillos, escobas y zunchos. Actualmente, procesos industriales de reciclado químico realizados bajo controles específicos de higiene y calidad permiten obtener R-PET factible de ser reutilizado en la fabricación de envases y botellas destinados a productos alimenticios. Las nuevas botellas admiten hasta un 51\% de R-PET cristal o celeste en su composición (Villavicencio, 2015). Este límite porcentual está determinado por la garantía de calidad en el nivel de color de la botella, ya que el PET se va degradando a medida que se lo reutiliza pudiendo afectar a largo plazo la presentación del envase y su contenido.

El disparador del presente estudio fue una entrevista con el área comercial de la empresa multinacional pionera en Argentina en la elaboración de envases para bebidas confeccionados con PET reciclado, la cual arrojó el siguiente dato: en nuestro país solo se recupera el $20 \%$ de los residuos reciclables, debido a la inexistencia de un sistema formal eficiente, que permita abastecer a las empresas del sector de suficiente material. 


\section{Metodología}

Según De Brito \& Dekker (2003), un estudio sobre reciclado debe centrarse en entender ¿por qué?, ¿qué?, ¿quién? y ¿cómo? ocurren los distintos procesos vinculados a una cadena de logística inversa. Para buscar respuesta a estos interrogantes hemos aplicado diferentes estrategias metodológicas:

- Consulta a fuentes primarias:

- Entrevistas personales o vía e-mail con los actores involucrados:

empresas, centros de acopio formales e informales, recuperadores urbanos.

- Sondeo de opinión para determinar el grado de conocimiento y la actitud de los vecinos.

- Consulta a fuentes secundarias:

- Análisis de literatura sobre gestión ambiental y logística inversa.

- Análisis documental de estudios, notas y noticias disponibles en Internet.

- Modelización de los caminos del PET en el mercado argentino con el Software Vensim PLE de Ventana Systems.

\section{Resultados y discusión}

El reciclaje de residuos en general y del PET en particular constituye en la actualidad una opción de negocios con buena recepción a nivel de la opinión pública por su relación directa con el cuidado ambiental. A esto se suma que en los últimos años se han desarrollado una serie de ventajas técnicas vinculadas a la actividad, como los detectores ópticos para la separación de plásticos, los sistemas de lavado automatizados, métodos de depuración del agua y los procesos de reciclado químico.

Dado que la materia prima para la industria del R-PET proviene de los residuos a priori podemos suponer que es posible disponer de un flujo de materiales constante y de muy bajo precio. Sin embargo, al estudiar la situación de esta actividad en el mercado argentino, hemos hallado que los siguientes puntos críticos dificultan la cadena de abastecimiento de las empresas recicladoras:

- Legislación insuficiente y de poco cumplimiento efectivo.

- Factores culturales: falta de motivación, estímulo y conocimiento para la separación en origen.

- Multiplicidad de actores involucrados en el proceso.

- Informalidad económica en las etapas intermedias de la recuperación.

- Dificultades logísticas para la obtención del PET usado, su transporte y acopio. 
El objetivo del estudio es realizar un análisis de estos puntos críticos que nos oriente a la hora de buscar soluciones que permitan optimizar la cadena de suministro del PET post consumo en Argentina.

\section{Análisis de puntos críticos}

\section{Legislación}

En Argentina, la legislación y normativa vinculada al tema de los RSU, su tratamiento y disposición final tiene como punto de partida lo expresado en el Artículo 41 de la Constitución Nacional: "Todos los habitantes gozan del derecho a un ambiente sano, equilibrado, apto para el desarrollo humano y para que las actividades productivas satisfagan las necesidades presentes sin comprometer las de las generaciones futuras; y tienen el deber de preservarlo. El daño ambiental generará prioritariamente la obligación de recomponer, según lo establezca la ley.” (Constitución Nacional, 1994).

En el ámbito nacional rige la Ley $\mathrm{N}^{0} 25.916$ (B.O. 7/09/04) de Protección Ambiental para la Gestión Integral de Residuos Domiciliarios, cuyos objetivos son lograr un adecuado y racional manejo de los residuos domiciliarios mediante su gestión integral, a fin de proteger el ambiente y la calidad de vida de la población y promover la valorización de los residuos domiciliarios a través de la implementación de métodos y procesos adecuados, minimizar los impactos negativos que estos residuos puedan producir sobre el ambiente y lograr la minimización de los residuos con destino a disposición final. Diversas leyes provinciales reproducen el espíritu de esta Ley Nacional, pese a lo cual hoy día en Argentina, aún son más los basurales a cielo abierto que los rellenos sanitarios.

Respecto a la gestión directa de los RSU, el nivel gubernamental más importante es el municipal, ya que es la instancia sobre la que recae la obligación de la recolección y disposición final. Es a este nivel donde se hace más evidente la disparidad de criterios y grados de aplicación. Existen ciudades, como Gualeguaychú y Buenos Aires, entre otras, donde es obligatoria la clasificación de los residuos, mientras que hay localidades, como Salta, que están estudiando su implementación. Sin embargo, en la mayoría de los casos donde existen normativas municipales sobre el tema, no se pueden cumplir en forma adecuada debido a la falta de contenedores para reciclables en la vía pública e inexistencia de recolección diferenciada por parte de los municipios. En estos casos, siendo las autoridades comunales las primeras en desatender la obligación de proveer los medios para cumplir las normativas, se justifica la infracción por parte de los vecinos, por lo que no se aplican las multas correspondientes. 


\section{Factores culturales, motivación y estímulo}

Una adecuada respuesta a la pregunta “iporqué retornar envases?” puede ser la fuerza motriz que de inicio a una cadena exitosa de reciclado del PET. La motivación se sustentará en diferentes argumentos, según se trate impulsar las acciones de las empresas o de los consumidores.

Según de Brito y Dekker (2003), las empresas se involucran en este tipo de programas 1) porque pueden obtener ganancias de ello. 2) porque tienen que hacerlo (razones legales) 3) porque se "sienten" socialmente motivadas a hacerlo. Las ganancias de las empresas pueden resultar de un beneficio directo obtenido al disminuir costos de producción o por un beneficio indirecto, ya que una estrategia "verde y sostenible" constituye hoy día una poderosa herramienta de marketing, útil para posicionar productos en determinadas franjas de mercado con buen poder adquisitivo. En consonancia, la "motivación social" de las empresas surge como necesidad de satisfacer a un mercado cada vez más exigente en cuestiones de responsabilidad ambiental.

Una vez que se produce la demanda por parte de las empresas, la cadena de reciclado del PET se apoya en los consumidores. La adecuada separación en origen facilita la recuperación del material. Los reciclables, preferentemente limpios y separados de los residuos orgánicos deben desecharse en los contenedores destinados a tal fin. Esto significa para los vecinos dedicar tiempo y esfuerzo a la realización de una tarea que bien podrían evitarse, descartando sus residuos sin clasificar y dejando que se ocupen de ellos los recuperadores urbanos.

Diversas experiencias indican que el reciclado y clasificación de los residuos por parte de los consumidores se estimula a través de dos mecanismos principales: 1) Campañas de difusión y educación ambiental. 2) Retribución económica.

Para analizar el impacto de las campañas de difusión y educación ambiental compararemos los resultados de un sondeo de opinión sobre residuos urbanos realizado por nuestro grupo de trabajo en el año 2009 y repetido en el año 2014 en la Ciudad de Buenos Aires y el Gran Buenos Aires. Entre ambas encuestas tuvo lugar la campaña de difusión y educación vinculada a la implementación de la ley Basura Cero, llevada adelante en medios locales y nacionales por el Gobierno de Buenos Aires.

El sondeo realizado en 2009 fue un estudio observacional, exploratorio, descriptivo y transversal sobre una muestra de 972 personas de ambos sexos, mayores de 18 años orientado a indagar la actitud de los vecinos respecto de varios aspectos vinculados a los RSU, entre ellos el reciclado. Frente a la pregunta ¿Clasifica la basura en su hogar? solo el $16 \%$ de los encuestados respondió afirmativamente. (López Sardi et al., 2009).

El estudio se repitió en el año 2014, sobre una muestra de 602 personas de ambos sexos. Frente a la misma pregunta, el 52\% aseguró que realiza algún grado de clasificación de la basura en su hogar (Figura 1): 


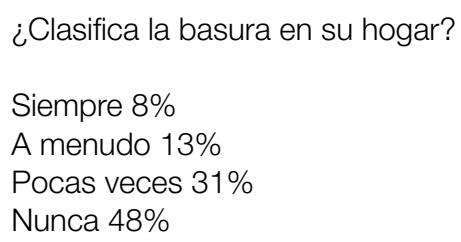

Figura 1. ¿Clasifica la basura en su hogar? Encuesta 2014.

En la edición 2014 de la encuesta el 63\% de los encuestados dijo conocer que los envases de PET causan contaminación ambiental.

Los resultados de la encuesta permiten observar un significativo avance en la actitud proactiva de los vecinos hacia la clasificación y el reciclado, motorizado por las campañas de difusión y educación ambiental denominadas "Ciudad Verde", que tuvieron lugar entre ambos sondeos. Ya son varios los municipios que han iniciado un proceso de instalación de contenedores para materiales reciclables, recolección diferenciada de residuos, apoyo a las cooperativas de recicladores urbanos y creación de puntos verdes.

\section{Acciones de motivación}

La retribución económica de los ciudadanos es la otra estrategia empleada para motivarlos a clasificar y separar en origen los RSU. Como ejemplo se detallan tres experiencias exitosas llevadas adelante en distintos países:

\section{Acopio Social (México):}

Básicamente el programa consiste en estimular el acopio de materiales reciclables por parte de los integrantes de comunidades de escasos recursos económicos. Quien entrega estos materiales en los centros verdes se hace acreedor a una serie de puntos mediante una tabla de equivalencias (ver Tabla 2), que luego pueden ser canjeados por alimentos de la canasta básica (ver Tabla 3). (Ecoce, 2015).

\begin{tabular}{|ll|}
\hline \multicolumn{2}{|c|}{ Equivalencias } \\
\hline $1 \mathrm{~kg}$ de PET & 3 puntos \\
\hline $1 \mathrm{~kg}$ de aluminio & 13 puntos \\
\hline $1 \mathrm{~kg}$ de multilaminado & 1 punto \\
\hline $1 \mathrm{~kg}$ de polietileno & 1 punto \\
\hline
\end{tabular}

Tabla 2. Puntos de equivalencia. 


\begin{tabular}{|c|c|c|c|}
\hline Aceite de $500 \mathrm{~mL}$ & 11 puntos & Jugo $450 \mathrm{~mL}$ & 6 puntos \\
\hline Agua $1,5 \mathrm{~L}$ & 7 puntos & Sal $1 \mathrm{~kg}$ & 6 puntos \\
\hline Arroz $1 \mathrm{~kg}$ & 11 puntos & Puré de tomates & 4 puntos \\
\hline Azúcar $1 \mathrm{~kg}$ & 11 puntos & Chiles en vinagre & 6 puntos \\
\hline Frijol $1 \mathrm{~kg}$ & 14 puntos & Lata de sardinas & 18 puntos \\
\hline Papel higiénico 4 rollos & 7 puntos & Harina de maíz $1 \mathrm{~kg}$ & 10 puntos \\
\hline Paquete de pasta & 3 puntos & Refresco $600 \mathrm{~mL}$ & 7 puntos \\
\hline
\end{tabular}

Tabla 3. Canje de alimentos por puntos.

Se benefician del programa más de 130.000 personas pertenecientes a 200 comunidades repartidas en 24 municipios de México. El programa lleva recuperados 954 millones de envases de PET, entre otros materiales. El aspecto que consideramos más destacable de esta experiencia es la capacidad de combinar responsabilidad ambiental y responsabilidad social. La asociación civil que lleva adelante la iniciativa está conformada por grupos industriales de productos alimenticios que proveen los alimentos que serán canjeados por los puntos y a su vez utilizan parte de los materiales recuperados en la campaña. El trabajo coordinado de la asociación con las autoridades fomenta la creación de la industria del reciclaje y promueve valores como el sentido de pertenencia a la comunidad, el autoempleo, la limpieza del medio ambiente, la educación ambiental y el sentido de colaboración.

\section{Viajes gratis de metro (Beijing, China):}

La compañía china de reciclaje INCOM instalará más de 100 máquinas recolectoras de envases PET en las estaciones de la línea 10 del metro de Beijing. El acuerdo entre la empresa y el gobierno permite que se canjeen los envases de refresco y agua vacíos por pasajes. Los transeúntes estarán recibiendo el equivalente a 15 centavos de dólar americano por cada botella introducida en las máquinas recicladoras. (Ecoosfera, 2013).

Consecución de un entorno limpio (Madrás, India):

EXNORA Internacional es una ONG de ciudadanos de Madrás preocupados por la gran cantidad de basura y suciedad presente en sus calles. Cuando las autoridades municipales de la ciudad decidieron en 1989 tomar medidas para enfrentar este problema, instalaron contenedores de basura en las esquinas y un sistema de recogida semanal de los contenedores. 
Ante la falta de colaboración de los vecinos, quienes seguían arrojando sus basuras en la calle, la asociación civil EXNORA, mediante un préstamo bancario compró carretillas de tres ruedas que entregó a los traperos (recicladores urbanos) junto con un sueldo de 650 rupias y el título honorario de "embellecedores de la calle". Estos recogen la basura y barren la calle. Un ingreso adicional que obtienen surge de la venta de los materiales reciclables a los chatarreros. Hoy día el programa se sostiene mediante el aporte de entre 1 y 20 rupias por parte de cada hogar ubicado en las calles beneficiadas. Actualmente existen en Madrás unos 750 centros EXNORA nucleando 75 familias por centro (Hábitat, 1996).

Como se observa en estos ejemplos, la oportunidad de obtener algún beneficio económico es un motor que garantiza compromiso y sobre todo continuidad por parte de las personas involucradas en el proceso de clasificación y recuperación de residuos.

\section{Actores implicados en el proceso}

La cadena de suministro de materias primas para la industria del R-PET se inicia en el momento en que se descarta una botella o envase usado y sigue hasta que, en las condiciones adecuadas, el PET que conforma esa botella o envase llega al sector industrial que lo reutilizará. El proceso de devolución, recuperación y reuso de envases PET involucra a una multiplicidad de actores, los cuales muchas veces desempeñan más de un rol en forma simultánea. Muchos de los actores habituales de la cadena operan al margen de la legalidad, en un sistema informal o "en negro" que se entrelaza con el sistema legal siendo aceptado con naturalidad y $\sin$ mayores cuestionamientos. La Figura 2 esquematiza en forma sencilla los principales actores de esta cadena de suministro y sus relaciones:

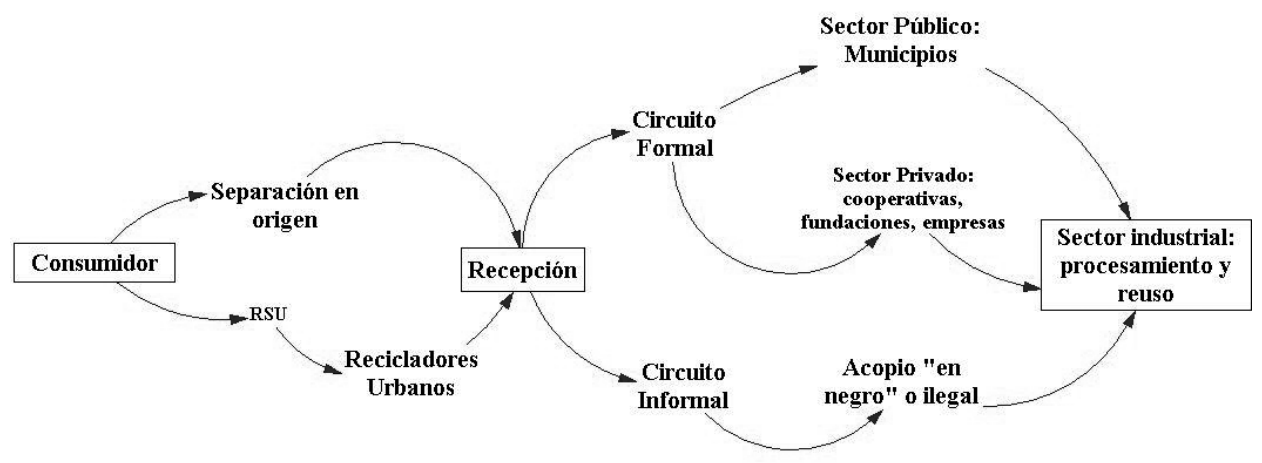

Figura 2. Actores del proceso. 


\section{Informalidad económica}

En Argentina, al no estar generalizada la obligación legal para la clasificación domiciliaria de residuos ni la recolección diferenciada en muchos municipios, gran parte de la separación y recuperación de los materiales reciclables es realizada a través de un circuito informal. Esta actividad se reproduce en toda América Latina constituyendo la base del sustento económico de muchas familias de escasos recursos. Se los identifica bajo múltiples denominaciones: pepenadores, recuperadores informales, cartoneros, chatarreros, traperos y recicladores informales entre otros. Este tipo de ocupación implica serios riesgos para la salud e integridad física de los trabajadores y desprotección social para sus familias acompañada en general de un elevado grado de discriminación (Jaiberth et al., 2009). Al realizar sus tareas sin asesoramiento técnico ni supervisión muchas veces contribuyen a aumentar el impacto ambiental de la basura, rompiendo las bolsas de residuos y desparramando el contenido en busca de aquellos materiales valiosos para comercializar.

En el ámbito de la República Argentina los puntos de recolección, centros verdes y plantas de clasificación oficialmente reconocidos constituyen una pequeña parte del negocio. La creación de cooperativas y asociaciones civiles constituye un camino para ir acercando en forma gradual la actividad del reciclado hacia la formalidad económica y laboral. En Argentina ya existen unas 25 organizaciones de este tipo, cuya finalidad es disminuir la desocupación e incrementar la inclusión social. Entre otras actividades funcionan como centros de recepción, separación, limpieza y acondicionado de materiales reciclables. Muchas de ellas ya están pasando a la etapa de implementar los equipos y tecnología que les permitan procesar los materiales recibidos para dar mayor valor agregado a los reciclados que luego colocan en el mercado. Las cooperativas tienen un total de 4200 trabajadores registrados. Sin embargo, durante nuestro estudio hemos notado que el segmento social dedicado a esta actividad de manera informal supera en número a los trabajadores registrados. Los circuitos en que este sector comercializa el producto de sus actividades crean un curioso entramado entre actividades legales e ilegales. Los precios de comercialización varían ampliamente de acuerdo a las condiciones del mercado y sus necesidades, y no se encuentran limitados por una ley que controle esta actividad comercial. La regulación del precio surge de un estimado intuitivo entre la cantidad mensual de recolección, la demanda del mercado del PET y la negociación y regateo entre el acopiador y el reciclador. A la falta de precio estándar de compra y venta, se suma la inexistencia de facturación u otros comprobantes y la evasión impositiva. Cuando estas variables hacen que el valor del PET baje temporariamente, los recicladores informales orientan su negocio a la recolección de otros materiales en su lugar. Esto causa grandes fluctuaciones en el precio y abastecimiento del PET post consumo. 
La Tabla 4 muestra un comparativo de los precios promedio que podía obtener un reciclador por $\mathrm{kg}$ de envases limpios de PET en CABA y GBA. Los datos fueron recabados durante el primer semestre de 2015 y están expresados en pesos argentinos (ARS).

\begin{tabular}{|l|c|c|c|}
\hline & Circuito informal & PyMEs & Grandes empresas \\
\hline PET cristal & ARS 2,00 & ARS $3,50+$ IVA & ARS 4,50 + IVA \\
\hline PET celeste & ARS 1,50 & ARS $2,50+$ IVA & ARS 2,90 + IVA \\
\hline PET verde & ARS 0,80 & ARS $2,00+$ IVA & ARS $2,75+$ IVA \\
\hline
\end{tabular}

Tabla 4. Comparativo de precios promedio por kg de PET post consumo.

El circuito informal, a pesar de ofrecer el menor precio es, paradójicamente, el que realiza el mayor volumen de negocios, dada la mayor cercanía y accesibilidad de estos puntos de acopio para los recicladores informales, por estar ubicados generalmente en barrios marginales o en los alrededores de los basurales. La no profesionalización de los recuperadores y acopiadores de este sector informal del mercado provoca que una parte del material así reunido se desperdicie, debido a falta de higiene y tratamiento inadecuado. Las empresas y los centros de acopio debidamente habilitados pagan mejor por kg de PET recuperado que el acopiador informal, pero tienen más altas exigencias en lo que a calidad, higiene y grado de separación se refiere. Compran únicamente materiales limpios, clasificados por tipo de código, densidad y/o color, sin etiquetas, sin polvo ni líquido ni restos de goma y adhesivos, entre otros requisitos.

La Cámara Argentina de Industrias de Reciclados Plásticos tiene registradas 56 empresas dedicadas a esta actividad. De ellas, solo 15 se ocupan del PET post consumo. La Tabla 5 sintetiza los resultados de nuestra investigación, acerca de los mecanismos de obtención de la materia prima empleados en cada empresa:

\begin{tabular}{|c|l|l|}
\hline Empresa & Fuente de abastecimiento & Producto final \\
\hline 1. & $\begin{array}{l}\text { Acopio mediante campañas en escuelas o } \\
\text { municipios. Retribución mediante obsequio } \\
\text { de mobiliarios plásticos a la institución }\end{array}$ & $\begin{array}{l}\text { Fábrica de mobiliario plástico } \\
\text { para hogar, jardín, etc. }\end{array}$ \\
\hline 2. & $\begin{array}{l}\text { Compra fardos de material limpio clasificado } \\
\text { por color o contenido previo. No especifica } \\
\text { quien es el proveedor. }\end{array}$ & $\begin{array}{l}\text { Escamas de PET en bolsones } \\
\text { de } 500 \mathrm{~kg}\end{array}$ \\
\hline 3. & $\begin{array}{l}\text { Compra material limpio proveniente de RSU. } \\
\text { No especifica el proveedor. }\end{array}$ & $\begin{array}{l}\text { Postes y varillas para } \\
\text { alambrados, tablas y tirantes } \\
\text { para carpintería. }\end{array}$ \\
\hline
\end{tabular}




\begin{tabular}{|c|c|c|}
\hline 4. & $\begin{array}{l}\text { Materias primas obtenidas de empresas, } \\
\text { lugares de desecho u otras compras a } \\
\text { terceros. }\end{array}$ & Material molido o pelletizado \\
\hline 5. & $\begin{array}{l}\text { PET cristal y celeste post industrial: botellas } \\
\text { y bidones de rechazo, preformas y tortas. } \\
\text { Trato directo con empresas. Recibe material } \\
\text { en big bag, limpio y sin prensar. }\end{array}$ & Escamas de PET de 4 a 10 mm \\
\hline 6. & $\begin{array}{l}\text { PET cristal verde o celeste. Origen: scrap } \\
\text { industrial, ya triturado. }\end{array}$ & $\begin{array}{l}\text { Separadores, cubetas y } \\
\text { bandejas para empaque de } \\
\text { fruta fresca. }\end{array}$ \\
\hline 7. & $\begin{array}{l}\text { Scrap industrial, material post consumo, } \\
\text { admite variados canales de abastecimiento: } \\
\text { empresas, acopiadores, plantas de } \\
\text { recuperación, escuelas, etc. Material } \\
\text { separado y limpio. }\end{array}$ & Pellets en bolsas de 25 kg. \\
\hline 8. & $\begin{array}{l}\text { PET cristal y verde post consumo } \\
\text { proveniente de RSU, a través de } \\
\text { acopiadores, recolectores informales, } \\
\text { iglesias, hogares, colegios, etc. }\end{array}$ & $\begin{array}{l}\text { PET enfardado. PET lavado y } \\
\text { molido. }\end{array}$ \\
\hline 9. & $\begin{array}{l}\text { PET post consumo de puntos verdes o } \\
\text { contenedores colocados en municipios } \\
\text { cercanos a la empresa. Material limpio y } \\
\text { clasificado. }\end{array}$ & $\begin{array}{l}\text { Mobiliario plástico, art. de } \\
\text { cámping, juguetes, accesorios } \\
\text { viales, postes, varillas par } \\
\text { alambrados, etc. }\end{array}$ \\
\hline 10. & $\begin{array}{l}\text { Material obtenido de empresas, municipios, } \\
\text { cooperativas de cartoneros y recicladores } \\
\text { informales. Material clasificado y enfardado. }\end{array}$ & Pellets y grumos. \\
\hline 11. & $\begin{array}{l}\text { Material proveniente de basurales obtenido a } \\
\text { través del circuito informal. }\end{array}$ & $\begin{array}{l}\text { Material molido, lavado, seco y } \\
\text { pelletizado. }\end{array}$ \\
\hline 12. & $\begin{array}{l}\text { Material obtenido de industrias locales, } \\
\text { vecinos y del circuito informal. Sin requisitos } \\
\text { de calidad. }\end{array}$ & $\begin{array}{l}\text { Bolsas, madera plástica, } \\
\text { postes y varillas, pallets y otros } \\
\text { productos plásticos. }\end{array}$ \\
\hline 13. & $\begin{array}{l}\text { Material obtenido en un } 90 \% \text { de acopiadores } \\
\text { informales, el resto vecinos, escuelas, etc. } \\
\text { Material clasificado y enfardado. }\end{array}$ & $\begin{array}{l}\text { Pellets, escamas de PET } \\
\text { verde y cristal en bolsones de } \\
500 \mathrm{~kg} .\end{array}$ \\
\hline 14. & $\begin{array}{l}\text { PET post industrial proveniente de purga } \\
\text { de máquinas, barrido de plantas, materiales } \\
\text { defectuosos, etc. }\end{array}$ & $\begin{array}{l}\text { Amplia gama de materias } \\
\text { plásticas y láminas. }\end{array}$ \\
\hline 15. & $\begin{array}{l}\text { Scrap industrial, limpio, clasificado y } \\
\text { enfardado. }\end{array}$ & Pellets. \\
\hline
\end{tabular}

Tabla 5. Fuentes de abastecimiento de las empresas argentinas de reciclado de PET.

De las 15 empresas dedicadas a la actividad en forma oficial, 8 de ellas (el 53\%) admiten recibir PET post consumo proveniente de canales informales de recolección.

Otro factor que distorsiona los precios del PET post consumo en el mercado Argentino es la dinámica de exportación de materiales a los grandes centros de 
reciclado ubicados en Asia, especialmente en China, que capta mayoritariamente el PET recuperado a nivel no solo nacional, sino también mundial.

\section{Dificultades logísticas:}

Si bien la dirección principal para los productos en el mercado es del productor hacia el consumidor final, se observa que es cada vez mayor el flujo de productos que "vuelven atrás". (de Brito \& Dekker, 2003). La correcta gestión de este flujo de materiales es estudiada por la logística inversa. Según Hawks (2006), logística inversa es el proceso de planificación, implantación y control, de forma eficiente y al coste óptimo, del flujo de materias primas y materiales en curso de producción y productos acabados, así como el de la información relacionada, desde el punto de consumo hacia el punto de origen con el objeto de recuperar el valor de los materiales o asegurar su correcta eliminación. Aplicando principios de logística inversa es posible lograr una gestión eficiente de materiales reciclables como el PET.

Una de las principales dificultades en la implementación de procesos de logística inversa para la recuperación de PET son las vinculadas al bajo peso y gran volumen de los envases a reciclar. Según García Olivares (2006), para reunir un kilogramo de PET son necesarias 33 botellas de refresco de $600 \mathrm{~mL}$ considerando unos $30 \mathrm{~g}$ por botella. Estamos hablando de aproximadamente $25 \mathrm{~m} 3$ / tonelada de botellas infladas. Esto constituye un grave problema cuando el material debe ser acopiado y luego transportado a la planta procesadora. El material sin compactar requiere grandes espacios para su almacenamiento y por su bajo costo, poco peso y gran volumen, no permite la recuperación de los costos del transporte requerido. Para resolver la situación generalmente se realiza el prensado/compactado del material en el punto de recepción o en el centro de acopio. Según Oviedo et al. , (2014), las plantas recicladoras deben ubicarse en locaciones cercanas a los puntos de generación, recepción y acopio de los materiales. La recomendación es situarlas en parques industriales cercanos a los centros urbanos. De este modo se simplifica la logística y se minimizan los costos de transporte.

Estas consideraciones contribuyen a explicar la persistencia en el negocio de los puntos de acopio del circuito informal (chatarrerías), ubicados a corta distancia de basurales y donde el traslado del material se realiza en carritos empujados por los propios recuperadores urbanos o cartoneros, sin gastos de combustible.

La Figura 2 representa los distintos caminos (directos e inversos) que recorre el PET en el mercado argentino. 


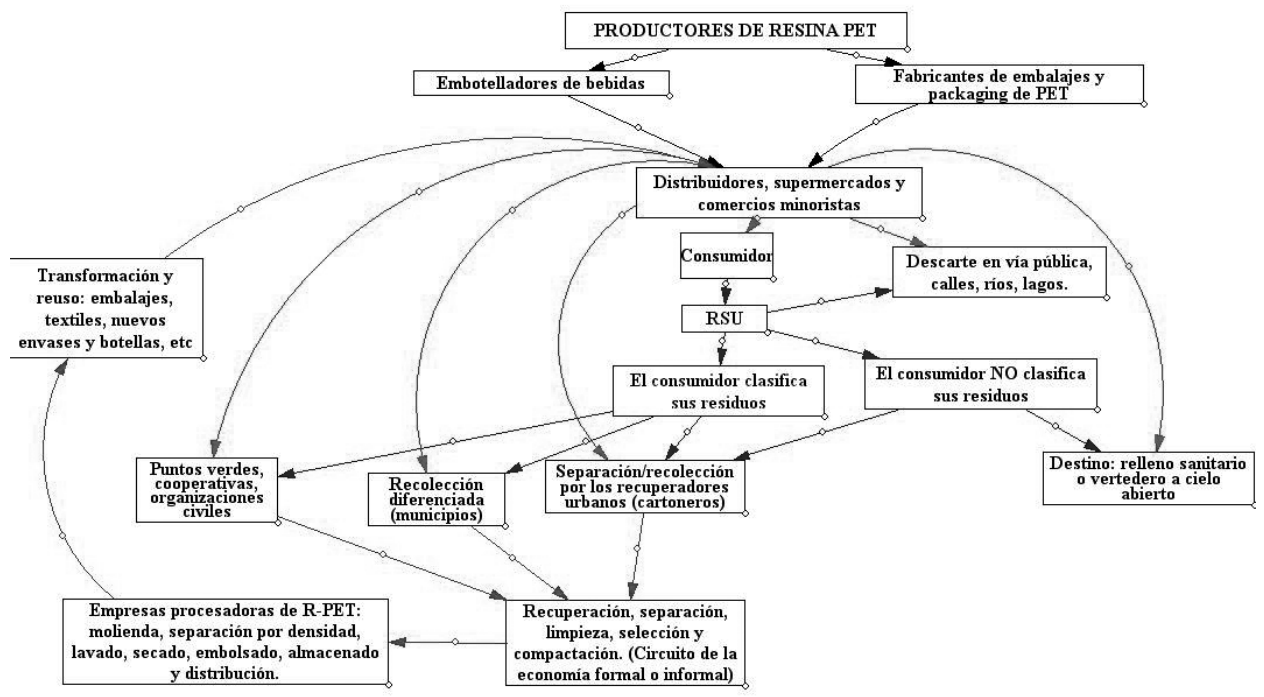

Figura 2. Caminos directos e inversos del PET en el mercado argentino.

El proceso está lejos de constituir un circuito de logística inversa eficiente, ya que muchos materiales que podrían reciclarse acaban en los rellenos sanitarios y vertederos o, peor aún, ensuciando la vía pública o arrojados en calles, ríos y lagos.

\section{Conclusiones}

Los emprendimientos vinculados al reciclado del PET constituyen una oportunidad de negocios interesante, especialmente desde que es posible su reutilización en packaging de alimentos y bebidas. La principal dificultad que enfrenta esta actividad en el mercado argentino está vinculada a la informalidad de la red de negocios tejida alrededor de la recuperación de los residuos y al desconocimiento y falta de motivación de la población en general.

Se impone la necesidad del ordenamiento de la actividad por parte de los distintos niveles gubernamentales. La legislación sobre clasificación, separación, reciclaje y disposición final de los residuos no alcanza si no está acompañada de la intención oficial de que esta legislación se cumpla. El cumplimiento debería comprometer principalmente a los propios municipios, efectivizando la presencia de contenedores verdes, la recolección diferenciada en sus comunas y el registro de todos los trabajadores, hoy informales, del sector. Una vez hecha su parte, las autoridades se verían con derecho a sancionar a aquellos que no cumplan con las disposiciones. 
Las campañas de educación ambiental y la retribución mediante pequeños beneficios económicos o sociales serían las herramientas adecuadas para vencer la resistencia de los vecinos a acatar la obligatoriedad de clasificar sus residuos.

Dar una solución integral a los puntos críticos de la cadena de suministro del PET post consumo requiere de un aporte multisectorial y será beneficioso para todos los actores involucrados en la actividad, porque permitirá disfrutar de un ambiente más limpio, con mejores oportunidades de trabajo y con nuevas perspectivas económicas.

\section{Fuentes consultadas}

Argentina. Ley 25916. Gestión de Residuos Domiciliarios. Boletín Oficial $N^{\circ}$ 30479 del 07-09-2004. Publicado el 22 de septiembre de 2004. . [en linea]. 2015. [Consultado julio de 2015]. Disponible en Web: <http://www.cairplas. org.ar/leyes/Ley_25916.pdf>

ANEP PET. Tecnología de transformación del PET. [en linea]. 2015. [Consultado julio de 2015]. Disponible en Web: <http://anep-pet.com/index.php/ conociendo-el-pet/procesos-de-transformacion>

Crawford, R.; Webb, H.; Arnott, J.; Ivanova, E. Plastic degradation and its environmental implications with special reference. [en linea]. Polymers. MDPI. [Basel. Switzerland]: 2013, 5,1 - 18. [Consultado julio de 2015]. Disponible en Web:

$<$ http://marinedebris.info/sites/default/files/literature/Plastic\%20Degradation $\% 20$ and $\% 20$ Its $\% 20$ Environmenta $\% 20$ Implications $\% 20$ with $\% 20$ Special $\% 20$ Reference\%20to\%20Poly(ethylene\%20terephthalate).pdf $>$ ISSN 2073-4360.

De Brito, M. P., Dekker, R. A framework for reverse logistics. [en linea]. ERIM Report Series Research in Management. [Rotterdam, The Netherlands]: 2003, 4. [Consultado agosto de 2015]. Disponible en Web: $<$ file:///C:/Documents $\% 20$ and\%20Settings/AIG/Mis\%20documentos/Downloads/SSRN-id423654.pdf>

Ecoce. Acopio Social. . [en linea]. 2015. [Consultado agosto de 2015]. Disponible en Web: < http://ecoce.mx/acopio-social.php >

Ecoosfera. Ciudadanos de Pekín pagan su viaje en metro con botellas de PET. [en linea]. 2013, 31 - 7. [Consultado agosto de 2015]. Disponible en Web: $<$ http://www.ecoosfera.com/2013/07/ciudadanos-de-pekin-pagan-su-viajeen-metro-con-botellas-de-pet/>

García Olivares, A.A. (2006) Recomendaciones táctico operativas para 
implementar un programa de logística inversa. Estudio de caso en la industria de reciclaje de plásticos. [en linea]. Biblioteca virtual de Derecho, Economía y Ciencias Sociales. Universidad de Málaga. [Málaga, España]. Ed. Eumed. net. 2006. [Consultado septiembre de 2015]. Disponible en Web: $<$ http://www. eumed.net/libros-gratis/2006a/aago/1d.htm>

Hawks, K. What is reverse logistics? [en linea]. VP Supply chain practice, Reverse Logistics Magazine [Main, USA]: 2006. [Consultado septiembre de 2015]. Disponible en Web: < http://www.rlmagazine.com/edition01p12.php>

Honorable Senado de la Nación Argentina. Constitución Nacional de la República Argentina. [en linea]. 1994. [Consultado julio de 2015]. Disponible en Web:

$<$ http://www.senado.gov.ar/Constitucion/capitulo1>

Jaiberth, A.; Cardona, A.; Díaz, A.; Elías, D.; López, A.; Yolanda, L. Autocuidado en recuperadores-recicladores informales de Medellin, Colombia, 2005. [en linea]. Revista Facultad Nacional de Salud Pública. Universidad de Antioquía. [Antioquía, Colombia]: 2009. [Consultado agosto de 2015]. Vol. 27. $\mathrm{N}^{\circ} 3$. Págs. 309-316. ISSN 0120-386X. Disponible en Web: <http://www.redalyc. org/articulo.oa?id=12016344008>

Junichiro, K. The $3 R$ Initiative. [en linea]. Policy planning división, waste, management and recycling. Ministry of the Environment. [Japón]: 2004. [Consultado julio de 2015]. Disponible en Web: < http://www.env.go.jp/ recycle/3r/en/info.html>

Lopes Francelino Goncalvez-Dias, S.; dos Santos de Souza Teodósio, A. Estrutura da cadeia reversa: caminos e descaminos da embalagem PET. [en linea]. Revista Producao, v. 16, n 3, p. 429 - 441. [San Pablo, Brasil]. 2009, 9 -12. [Consultado septiembre de 2015]. Disponible en Web:

$<$ http://www.scielo.br/scielo.php?pid=S0103-65132006000300006\&script=sci arttext>

López Sardi, M. Diez, S.M. Los vecinos y la gestión de residuos urbanos. Revista Empresas, productos y servicios, ambiente y saneamiento. Ed. AIDIS Argentina. Págs. 18 a 28. [Buenos Aires, Argentina]: 2009. ISSN 18515770.

Observatorio Nacional para la Gestión de Residuos Sólidos Urbanos. Generación. [en linea]. Secretaría de Ambiente y Desarrollo Sustentable de la Nación. [Buenos Aires, Argentina]: 2009. [Consultado agosto de 2015]. Disponible en Web: <http://www.ambiente.gob.ar/observatoriorsu/informacion_general/ en_arg_generacion.html> 
Oviedo, T.J.; Cuozzo, J.D. Estudio de factibilidad para planta de reciclado de residuos de plástico PET. [en linea]. p. 59. Universidad Nacional de Córdoba. Facultad de ciencias exactas, Físicas y Naturales. Escuela de Ingeniería Industrial. [Córdoba, Argentina]: 2014. [Consultado octubre de 2015]. Disponible en Web:

$<$ https://rdu.unc.edu.ar/bitstream/handle/11086/1542/Proyecto\%20Integrador\%20 -\%20Estudio\%20de\%20Factibilidad\%20para\%20Planta\%20de\%20Reciclado\%20 de $\% 20$ Residuos $\% 20 \mathrm{de} \% 20 \mathrm{Pl} \% \mathrm{C} 3 \%$ A 1 stico $\% 20$ PET.pdf?sequence $=1>$

Pilato, L., Weber, M., Kowatsch, S., Valdez, D., et al., Phenolic Resins: A century of Progress. Springer-Verlag [en linea]. p. 518. Springer. [Berlin Heidelberg]: 2010. [Consultado julio de 2015]. Disponible en Web:

http://link.springer.com/book/10.1007\%2F978-3-642-04714-5 ISBN 978-3-64204714-5.

Sancho Martín, C. Polietilentereftalato. [en linea]. 2015. [Consultado julio de 2015].

Disponible en Web: < http://www.eis.uva.es/ macromol/curso04-05/pet/ >

Tecnología de los plásticos. PET. [en linea]. 2011, 30-5. [Consultado julio de 2015]. Disponible en Web: <http://tecnologiadelosplasticos.blogspot.com. ar/2011/05/pet.html>

Villavicencio. Rebotella. Danone. [en linea]. 2015. [Consultado julio de 2015]. Disponible en Web: < http://www.villavicencio.com.ar/infografia.html> 